\title{
Urothelial Carcinoma: Highlights and Reviews on Various Pathologies
}

\author{
Authors: \\ Brian Dick, Olayemi Olubowale, Joseph Kim, *Spencer Krane \\ Department of Urology, Tulane University School of Medicine, New Orleans, Louisiana, \\ USA \\ *Correspondence to Lkrane1@tulane.edu \\ Disclosure: $\quad$ The authors have declared no conflicts of interest. \\ Received: $\quad 17.12 .19$ \\ Accepted: $\quad 06.03 .20$ \\ Keywords: $\quad$ Bladder cancer, differentiation, histological variants, urothelial carcinoma. \\ Citation: $\quad$ EMJ Urol. 2020;8[1]:46-53.
}

\begin{abstract}
Bladder cancers are the ninth most frequently diagnosed cancer worldwide. More than $90 \%$ of bladder cancers are of transitional cell origin and are classified as urothelial carcinomas (UC). UC remains amongst the most genetically diverse tumours and presents with numerous unique histological variants. The most common variants are squamous differentiated UC and glandular differentiated UC. Both of these variants tend to present at a later disease stage than conventional UC and are associated with worse patient outcomes. Rarer UC variants include trophoblastic differentiated UC, nested UC, micropapillary UC, plasmacytoid UC, and sarcomatoid UC. They also present at more advanced disease states than conventional UC, resulting in worse patient outcomes. Limited data is available for the pleomorphic giant cell UC and lipid-rich UC variants, but it suggests morbid outcomes with high patient mortality. The only UC variant with better prognosis than conventional UC is lymphoepithelioid-like UC. Proper identification of the histological variant of UC is important, as it aids the physician in clinical decision-making and can lead to better patient outcomes.
\end{abstract}

\section{INTRODUCTION}

The American Cancer Society estimates that there will be 80,470 new diagnoses of bladder cancer and 17,670 deaths from bladder cancer in the USA during 2019. It is the ninth most frequently diagnosed cancer worldwide, of which the highest incidence is observed in men. ${ }^{2}$ Most bladder cancers are epithelial in origin, and $>90 \%$ are classified as urothelial (transitional) carcinoma (UC). ${ }^{3}$ UC has a proclivity to differentiate, and the 2016 World Health Organization (WHO) Classification of Tumors of the Urinary System has identified many histological variants (Table 1). ${ }^{4}$ The mainstay of treatment for

muscle-invasive urothelial malignancy remains neoadjuvant chemotherapy followed by radical cystectomy; however, identifying the proper UC histological variant can guide treatment and provide more accurate prognoses. Urothelial cancer remains amongst the most highly genetically diverse tumours, which leads to substantial variation in histology. ${ }^{5}$ With so many variant histologies, many cases require evaluation by a specialised genitourinary pathologist. ${ }^{6}$ In this review, the identifying features of common UC histological variants and the expected patient prognoses are discussed. This is not a systematic review of the literature but a highlight of most common variants. 


\begin{tabular}{|l|}
\hline \multicolumn{1}{|c|}{ Urothelial Tumours } \\
\hline Urothelial carcinoma with divergent differentiation \\
Squamous differentiation \\
Glandular differentiation \\
Trophoblastic differentiation \\
Nested urothelial carcinoma \\
Micropapillary urothelial carcinoma \\
Lymphoepitheliod-like urothelial carcinoma (LELUC) \\
Plasmacytoid urothelial carcinoma \\
Sarcomatoid urothelial carcinoma \\
Pleomorphic giant cell urothelial carcinoma (PGCUC) \\
Lipid-rich urothelial carcinoma
\end{tabular}

Table 2: Studies comparing outcomes in squamous differentiation and stage-matched pure urothelial carcinoma.

\begin{tabular}{|l|l|l|l|l|}
\hline Study & $\begin{array}{l}\text { Patients with } \\
\text { squamous } \\
\text { differentiation }\end{array}$ & Cancer stages & Treatment & $\begin{array}{l}\text { Significant difference } \\
\text { in overall survival? }\end{array}$ \\
\hline $\begin{array}{l}\text { Nishiyama et al., } \\
2004\end{array}$ & 38 & All & All & RC \\
\hline Xylinas et al., ${ }^{12} 2013$ & 227 & All & RC & No \\
\hline Monn et al., ${ }^{19} 2015$ & 68 & PT1 & RC & No \\
\hline Li et al., ${ }^{20} 2017$ & 227 & MIBC & TURBT & Yes \\
\hline Sefik et al., ${ }^{14} 2018$ & 17 & MIBC & RC & No* \\
\hline Minato et al., ${ }^{15} 2018$ & 20 & MIBC & RC & Yes \\
\hline Marks et al., ${ }^{21} 2018$ & 62 & RC & No \\
\hline
\end{tabular}

*There was a significant difference in postoperative tumour stage and upstaging was more likely. MIBC: muscle invasive bladder cancer; PT1: primary tumour Stage 1; RC: radical cystectomy; TURBT: transurethral resection of bladder tumour.

\section{UROTHELIAL CARCINOMA WITH DIVERGENT DIFFERENTIATION}

UC with divergent differentiation is the most common variant of UC. The 2016 World Health Organization (WHO) classification lists subtypes for squamous, glandular, and trophoblastic differentiation. ${ }^{4}$ It is recommended that the percentage of differentiated tissue be included in the pathology report.

\section{Squamous Differentiation and Variants}

Squamous differentiation is the most common subtype of UC with divergent differentiation and occurs in $14-40 \%$ of all cases of UC.,7,8 It is defined by the presence of intracellular bridges and keratinisation. ${ }^{4}$ There is an association between human papillomavirus and squamous differentiation but the role of the virus in tumourigenesis is unclear. $^{9}$ Immunohistochemical markers for squamous differentiation include p63 (100\%), HMCK (100\%), CK14 (87\%), CK7 (80\%), S10OP (78\%), thrombomodulin (70\%), desmoglein-3 (70\%), GATA3 (35\%), and uroplakin 
III (13\%). 10,11 The molecular subtype of squamous differentiated UC is basal. ${ }^{6}$

It is widely reported that squamous differentiation is significantly more likely to present with advanced tumour stage, lymph node metastasis, and lymphovascular invasion when compared to pure UC. ${ }^{12-17}$ However, it is unclear if patients with squamous differentiation have a worse prognosis than patients with stage-matched pure UC. ${ }^{12,14,15,18-21}$ Table 2 shows studies that examine overall survival in patients with squamous differentiation compared to patients with pure UC, and demonstrates inconsistent results. The comparison of squamous differentiation to stagematched pure $U C$ is interesting from an academic perspective but holds less weight for patients. Squamous differentiation typically presents at a later disease stage, which is associated with a worse outcome.

Partial sampling of squamous differentiation or poorly demarcated squamous differentiation can lead to erroneous classification as pure squamous cell carcinoma. ${ }^{22}$ To further blur the lines, the two cancers are histochemically similar. The main difference is the $0 \%$ expression of GATA3 in pure squamous cell carcinoma compared to $35 \%$ expression in squamous differentiation." Other aspects of a patient's history are usually required to aid diagnosis. For example, pure squamous carcinoma comprises $1.3 \%$ of bladder carcinomas in Western countries, but makes up $60.0 \%$ of cases where schistosomiasis is endemic. ${ }^{23}$ In ambiguous cases, patients are given a diagnosis by a multidisciplinary team based on clinical history and the presence or absence of a clear-cut conventional UC component.22,24 With increasing options for treatment, including immunooncologic therapies along with conventional chemotherapy, further studies will hopefully be directed at identifying the most effective systemic therapy option in these patients.

\section{Glandular Differentiation and Variants}

Glandular differentiation is the second most common subtype of UC with divergent differentiation. ${ }^{4}$ It is estimated to comprise $6-18 \%$ of cases of UC. ${ }^{6}$ Glandular differentiation is defined by the presence of glands within UC. The enteric variant of this tumour is easily confused with colonic adenocarcinoma as they may present similarly. Particularly in transurethral resection of bladder tumour (TURBT) specimens arising from the posterior of the bladder or with vesicoenteric fistulae, it can be unclear from which organ the primary malignancy arises. The mucinous variant presents as cells (occasionally signet ring-like cells) floating in extracellular mucin.4,6,24 Immunohistochemical markers for glandular differentiation include S10OP (100\%), CK2 (100\%), CK7 (90\%), HMCK (90\%), p63 (60\%), GATA3 (50\%), and uroplakin III (10\%). " The molecular subtype of glandular differentiated UC is luminal. ${ }^{6}$

There is limited data regarding prognosis for individuals with glandular differentiation; most studies combine patients with glandular differentiation, squamous differentiation, or both, into one group. When compared to stagematched pure UC, glandular differentiation + - squamous differentiation is significantly more likely to present with lymphatic spread at time of diagnosis and extravesical disease at time of cystectomy. ${ }^{16,17,25}$ However, the data is variable regarding overall survival in glandular differentiation +/- squamous differentiation compared to stage-matched pure UC. Wasco et al. ${ }^{16}$ and Kim et al. ${ }^{17}$ examined patients with any stage glandular differentiation +/- squamous differentiation who had received either a TURBT or radical cystectomy and found no difference in overall survival compared to stage-matched pure UC. Xu et al..$^{25}$ looked specifically at patients who received TURBT and chemotherapy for nonmuscle invasive bladder cancer with glandular differentiation +/- squamous differentiation and found that glandular differentiation +/- squamous differentiation was an independent predictor of recurrence-free survival, but not progression-free survival. In the only study looking specifically at glandular differentiation, Zhao et al. ${ }^{26}$ reported that primary tumour Stage 1 (pT1) glandular differentiation patients have significantly higher recurrence and progression rates compared to stage-matched pure UC. They recommend radical cystectomy in recurrent cases.

\section{Trophoblastic Differentiation}

Trophoblastic differentiation is a rarer subtype of UC with divergent differentiation. It is defined by the presence of syncytiotrophoblasts, which make human chorionic gonadotrophin (hCG). ${ }^{4}$ The syncytiotrophoblasts often have multiple, 
large nuclei and an eosinophilic cytoplasm with cytoplasmic lacunae. Immunohistochemical markers for trophoblastic differentiation include the $\beta$-subunit of hCG, placental alkaline phosphatase, human placental lactogen, a-inhibin, epithelial membrane antigen, and various $\mathrm{CK}$ $(1-8,10,14-16,19) .^{27}$ The molecular subtype of trophoblastic differentiated UC is unknown. ${ }^{6}$

Due to its rarity, there is little information regarding prognosis for trophoblastic differentiation. Elevated hCG levels may be associated with poor response to radiation therapy and increased rate of disease progression. Survival time is $<1$ year in most cases. ${ }^{27}$

\section{NESTED UROTHELIAL CARCINOMA AND VARIANTS}

Nested UC has been reported in patients aged 42-90 years, but has a propensity for men $>60$ years-old. ${ }^{28}$ The 2016 WHO classifications characterise nested UC as cytologically bland tumour cells which infiltrate as disorderly, discrete, or confluent nests or tubules. ${ }^{4}$ The nests are closely packed and have an irregular shape and distribution. ${ }^{23}$ The key to identifying nested $U C$ is understanding its infiltrative nature and recognising the irregular epithelial-stromal interface that indicates invasion. ${ }^{3}$ Deeper portions of a tumour tend to have more advanced cellular atypia. ${ }^{23}$ The 2016 WHO classifications go on to describe a 'large variant' of nested UC, which is not mentioned in the 2004 WHO classifications. ${ }^{4}$ Compared to typical nested UC, the large variant has bigger cell nests and more fibrous stromal tissue between nests. ${ }^{27}$ Immunohistochemical stains for nested UC include CK7 (100\%), p63 (100\%), HMCK (100\%), S10OP (90\%), GATA (70\%), thrombomodulin (55\%), uroplakin III (40\%), and CK2O (30\%). ${ }^{11}$ The molecular subtype of nested UC can be either luminal or basal. ${ }^{6}$

Nested UC tends to present with advanced tumour stage and lymph node invasion. ${ }^{23,24}$ Compared to conventional UC, nested UC is associated with a higher incidence of metastatic disease, muscle invasion at TURBT, and extravesical disease at cystectomy. ${ }^{29}$ At similar stages, there is similar survival to conventional UC. Linder et al. ${ }^{30}$ identified 52 nested UC patients treated with radical cystectomy and found no increased rate of disease recurrence or adverse survival compared to patients with stage-matched conventional UC. Mally et al. ${ }^{31}$ identified 30 non-muscle invasive nested UC patients treated with TURBT and found that nested UC metastasises to lymph nodes earlier and presents with a higher rate of upstaging when compared to non-muscle invasive conventional UC patients. However, no significant difference in metastasisfree survival or cancer-specific survival was found. Based on this limited data, early radical cystectomy may be beneficial in patients presenting with non-muscle invasive nested UC.

\section{MICROPAPILLARY UROTHELIAL CARCINOMA AND VARIANTS}

Micropapillary UC is an aggressive variant of UC with an estimated prevalence of $0.7-8.0 \% .^{24}$ The mean age of presentation is 66 years. ${ }^{27}$ According to the 2016 WHO classifications, micropapillary UC is morphologically characterised by small nests and aggregates of tumour cells within lacunae. ${ }^{4}$ Surface tumours are characterised by filiform processes and papillary tufts, and they may also contain fibrovascular cores. Invasive tumours are often seen in tissue retraction spaces and contain small, tight nests of cells. ${ }^{27}$ Tumours that only have micropapillary UC morphology in the surface component are not associated with worse outcomes compared to conventional UC. Accordingly, classification of micropapillary $U C$ requires the invasive component of the tumour to show micropapillary morphology. ${ }^{6}$ Immunohistochemical stains for micropapillary UC include epithelial membrane antigen, CK2O, Leu-M1, CK7 (100\%), S10OP (96\%), HMCK (96\%), GATA3 (86\%), CK2 (73\%), p63 (54\%), uroplakin III (38\%), and thrombomodulin (38\%). ${ }^{11,27}$ The molecular subtype of micropapillary UC is luminal in $>50 \%$ of cases. ${ }^{6}$

The prognosis for micropapillary UC is poor; $20 \%$ of patients present with metastases at time of diagnosis. ${ }^{27}$ Some suggest that patient prognosis is linked to the proportion of micropapillary UC within a tumour, but this concept remains controversial.6,22,23,27 When standard clinicopathologic predictors are controlled for, there is no significant difference in long-term outcomes following radical cystectomy in micropapillary UC patients compared to conventional UC patients. ${ }^{32}$ Treatment of 
micropapillary UC with bacillus Calmette-Guérin therapy is prone to failure, with many patients going on to develop metastases and few surviving longer than 10 years. ${ }^{33}$ A 2019 metaanalysis reviewed treatment outcomes in 3,154 patients with tumour Stage 1 (T1) micropapillary UC and found that while the 5-year cancerspecific survival for TURBT or bacillus CalmetteGuérin therapy was only $60-85 \%$, it was $81-100 \%$ for early radical cystectomy. ${ }^{34}$ Early cystectomy is generally preferred in this subset of patients.

\section{LYMPHOEPITHELIOID-LIKE UROTHELIAL CARCINOMA AND VARIANTS}

Lymphoepithelioid-like urothelial carcinoma (LELUC) of the bladder represents between 0.4$1.3 \%$ of all bladder cancers and has been reported in patients aged 44-90.35,36 Histologically, LELUC has a mixed epithelial composition on a background of inflammatory infiltrate. ${ }^{37}$ This infiltrate is normally lymphocyte-dominated but can also be composed of an assortment of neutrophils, eosinophils, lymphocytes, histiocytes, and plasma cells. ${ }^{38}$ The mixed epithelial cells form syncytial-like sheets, have prominent nucleoli, and undergo brisk mitotic activity. ${ }^{39}$ Despite LELUC having a similar appearance to nasopharyngeal carcinoma, it is not associated with EpsteinBarr virus (EBV) infection. ${ }^{40}$ Various stains are used to demonstrate both the epithelial (CK7, CK20, AE1, AE3, EMA, CD46v6) and lymphocytic (CD20, CD21, CD45RO, CD68, CD79a, D33) tumour components. ${ }^{41}$

The scarcity of reported LELUC cases has caused difficulty in defining prognosis and optimum treatment. ${ }^{35}$ Several studies have shown that tumours which consist predominantly or purely of LELUC carry a better prognosis than those with only a focal LELUC component. When focally present, the neoplasm behaves like conventional UC of similar grade and stage. ${ }^{36,42}$ A pooled analysis of 56 patients concluded that predominant or pure LELUC is amenable to bladder-preserving treatments while focal disease requires radical cystectomy. ${ }^{35}$ In the aforementioned study, patients with predominant or pure LELUC that received systemic chemotherapy following TURBT demonstrated a 100\% disease-free survival at a median follow-up of 34 months, while patients who received TURBT without systemic chemotherapy had 53\% disease-free survival at a median follow-up of 25 months. Platinum-based agents have shown encouraging outcomes; cisplatin was utilised as a primary chemotherapy for three patients with muscle-invasive bladder LELUC, and all three remained free of recurrence after 6 years follow-up. ${ }^{35}$

\section{PLASMACYTOID UROTHELIAL CARCINOMA AND VARIANTS}

Plasmacytoid UC tumours are uncommon, although the incidence of plasmacytoid differentiation in muscle-invasive bladder cancer was found to be $2.7 \% .{ }^{43}$ The age of reported cases ranges from 48-87 years. ${ }^{27}$ Plasmacytoid differentiation consists of histologically dissociated cells with eccentrically placed or irregular nuclei, eosinophilic cytoplasms, and occasional eosinophilic perinuclear halos (reminiscent of plasma cells). On top of this plasma cell morphology, single cells with cytoplasmic vacuoles (with or without mucin) impart a signet ring appearance. ${ }^{6}$ Loss of epithelial-cadherin, encoded by $C D H 1$, has been described in a large cohort of plasmacytoid UC patients, and may account for the decohesion of cells. ${ }^{6}$ This infiltration mechanism allows it to permeate the bladder in a linitis plastica-like manner, spreading along fascial planes into the peritoneum, and a 'coat sleeve pattern' along nerve bundles. ${ }^{44}$ In tracking a carcinoma capable of such spread, it is important to note that antibodies for CK7 and CK2O confirm this cancer's epithelial origin, and a majority of cells label for CK AE1/AE3, EMA, GATA3, CD15, p53, and p16. ${ }^{3}$ Multitarget fluorescence in situ hybridisation has shown plasmacytoid UC tumours to be both aneuploid and polysomic, with deletions on chromosome 9p21 being common. ${ }^{27}$

Plasmacytoid UC has characteristic infiltration that causes it to present with higher tumour stage and greater metastases than conventional UC. It is more likely to be associated with positive surgical margins. ${ }^{6}$ Its extensive bladder wall involvement and extension into perivesical soft tissue leads to increased rates of local recurrence, metastatic disease, and cancer-related deaths compared to conventional UC. ${ }^{45}$ A diagnosis of plasmacytoid UC carries a poor prognosis; hence, correct diagnosis is essential. Immediate radical cystectomy is typically suggested. ${ }^{33}$ 


\section{SARCOMATOID UROTHELIAL CARCINOMA AND VARIANTS}

Sarcomatoid UC comprises $0.3-0.6 \%$ of all bladder cancers. 3,33 The mean age and range of patient ages is 60 and $50-77$ years, respectively. ${ }^{46}$ These tumours have carcinomatous and sarcomatous components when observed histologically and immunohistochemically.,6 Histologically, sarcomatoid UC displays anaplastic spindle cells densely compacted into bundles. ${ }^{3}$ The immunoprofile of sarcomatoid UC includes staining for p63 (69\%), HMCK (56\%), CK7 (56\%), GATA3 (30\%), S10OP (27\%), CK20 (6\%), PAX8, and CK AE1/AE3.11,27 Molecular data shows that the carcinomatous and sarcomatous components are derived from the same malignant clone. ${ }^{3}$

Sarcomatoid UC is highly malignant, has poor prognosis, and tends to metastasise widely. ${ }^{3}$ A 2014 National Cancer Database study of 489 patients showed that sarcomatoid UC most commonly presents at clinical tumour Stage 2 (cT2) disease and has a median survival of 18.4 months. ${ }^{47}$ TERT C228T mutations are present in $35 \%$ of sarcomatoid UC, and all patients with this mutation died of cancer within 2 years of surgery. ${ }^{6}$ Even shorter overall survival may be associated with carcinomas that have myxoid or choroid features. ${ }^{24}$ Early cystectomy is recommended for sarcomatoid UC due to higher cancer-specific mortality compared to patients with conventional UC. ${ }^{33}$ High incidence of local and distant metastases necessitate the use of follow-up adjuvant chemotherapy and radiation in most patients. ${ }^{48}$ Further multicentre research is necessary to establish adequate treatment recommendations. ${ }^{48}$

\section{PLEOMORPHIC GIANT CELL UROTHELIAL CARCINOMA AND VARIANTS}

Pleomorphic giant cell urothelial carcinoma (PGCUC) is a rare, aggressive form of bladder cancer that is similar in morphology to giant cell carcinoma of the lung. Its features were not delineated until 2009. ${ }^{49}$ PGCUC is composed of giant, anaplastic, and bizarre multinucleated cells that oftentimes form nests, but can also arrange into cords which infiltrate the muscular wall of the bladder. ${ }^{3}$ PGCUC occurs more frequently in older males and has been reported in patients aged 55-88 years. ${ }^{27}$ Immunohistochemically, PGCUC stains positive for CK7, anti-CK (CAM 5.2), EMA, p63, and GATA3; the literature notes that this means immunohistochemistry plays a limited role in identification. ${ }^{6}$

PGCUC typically presents in combination with other types of UC. In a study of 13 patients with PGCUC, eight had high-grade UC, five had UC in situ, three had micropapillary UC, and one had plasmacytoid UC. ${ }^{50}$ PGCUC tends to have lymph node metastases at time of presentation and overall prognosis is poor. ${ }^{3}$ In a study of 10 patients by Samaratunga et al., ${ }^{50}$ five died within 1 year, one developed metastases at 17 months, three had recurrent high-grade disease within 3 years, and only one was alive and well at 46-month followup. A separate study reported on a group of eight patients with PGCUC; seven of the patients were either dead or alive with metastases at 2-year follow-up, and only one patient was alive and well. ${ }^{49}$ Multiple studies suggest that PGCUC represents an extreme de-differentiation of conventional UC, which explains why prognosis is so poor. ${ }^{49,50}$ Total excision is an adequate treatment choice. ${ }^{51}$

\section{LIPID-RICH UROTHELIAL CARCINOMA AND VARIANTS}

Lipid-rich UC is a rare tumour that has presented in patients aged 42-92 years, with the mean age being $70 .{ }^{27}$ Histologically, lipid-rich UC is comprised of lipoblast-like cells with eccentrically placed nuclei and abundant vacuolated cytoplasms. ${ }^{6}$ Immunohistochemical stains for lipid-rich UC include CK AE1/AE3 and CK7, and variably include CK2O, CAM 5.2, EMA, thrombomodulin, and CK34BE12. ${ }^{27}$ Molecular analysis has revealed a loss of heterozygosity patterns at polymorphic microsatellite marker sites DS9S171, D9S177, IFNA, and TP53; this suggests a common clonal origin between conventional UC and lipid-rich UC. 6,27,52

Most cases of lipid-rich UC present at an advanced stage and it may be associated with worse outcomes. ${ }^{6}$ In the largest study to date, Lopez-Beltran et al. ${ }^{6}$ reported on 27 cases;;2 $40 \%$ of patients had lymph node metastases at time of presentation. At 58-month follow-up, 16 patients had died of disease, three died of other causes, and eight patients were alive with the disease. As 
there is a limited number of published cases of lipid-rich UC, therapeutic strategies to treating this cancer are unclear. ${ }^{24}$

\section{CONCLUSION}

There are many histological variants of UC. Correct identification, while difficult, allows physicians to more accurately inform patients of their prognoses. Lymphoepithelioid-like UC is the only variant that holds a more benign prognosis than conventional UC. Squamous differentiated trophoblastic glandular differentiated, micropapillary, plasmacytoid, and sarcomatoid UC all present at more advanced disease states than conventional UC, resulting in worse patient outcomes. Pleomorphic giant cell UC and lipidrich UC are rarer disorders but the limited data available indicates high patient mortality. Early identification of the more malignant variants of UC is important as it aids the physician in clinical decision-making and can lead to better patient outcomes.

\section{References}

1. Siegel RL et al. Cancer statistics, 2019. CA Cancer J Clin. 2019;69(1):7-34.

2. Antoni $\mathrm{S}$ et al. Bladder cancer incidence and mortality: a global overview and recent trends. Eur Urol. 2017;71(1):96-108.

3. Damjanov I, Golubovic $M$. Histopathology of urinary bladder carcinoma: less common variants. Srp Arh Celok Lek. 2011;139(9-10):693-9.

4. Humphrey PA et al. The 2016 WHO classification of tumours of the urinary system and male genital organs-part B: prostate and bladder tumours. Eur Urol. 2016;70(1):106-19.

5. Lawrence MS et al. Mutational heterogeneity in cancer and the search for new cancer-associated genes. Nature. 2013;499(7457):214-8.

6. Lopez-Beltran A et al. Variants and new entities of bladder cancer. Histopathology. 2019;74(1):77-96.

7. Gluck $\mathrm{G}$ et al. Comparative study of conventional urothelial carcinoma, squamous differentiation carcinoma and pure squamous carcinoma in patients with invasive bladder tumors. J Med Life. 2014;7(2):211-4.

8. Kucuk $U$ et al. Clinical, demographic and histopathological prognostic factors for urothelial carcinoma of the bladder. Cent European J Urol. 2015;68(1):30-6.

9. Guma S et al. Papillary urothelial carcinoma with squamous differentiation in association with human papilloma virus: case report and literature review. Am J Clin Exp Urol. 2016;4(1):12-6.

10. Gulmann $\mathrm{C}$ et al. Immunohistochemical profile to distinguish urothelial from squamous differentiation in carcinomas of urothelial tract. Hum Pathol. 2013;44(2):164-72.

11. Paner GP et al. Immunohistochemical evaluation of novel and traditional markers associated with urothelial differentiation in a spectrum of variants of urothelial carcinoma of the urinary bladder. Hum Pathol. 2014;45(7):1473-82.

12. Xylinas $E$ et al. Impact of histological variants on oncological outcomes of patients with urothelial carcinoma of the bladder treated with radical cystectomy. Eur J Cancer. 2013;49(8):1889-97.

13. Liu $Y$ et al. Urothelial carcinoma with squamous differentiation is associated with high tumor stage and pelvic lymph-node metastasis. Cancer Control. 2017;24(1):78-82.

14. Sefik E et al. Effect of variant histology presence and squamous differentiation on oncological results and patient's survival after radical cystectomy. Arch Ital Urol Androl. 2018;90(3):172-5.

15. Minato $A$ et al. Clinical significance of squamous differentiation in urothelial carcinoma of the bladder. Cancer Control. 2018;25(1):1073274818800269.

16. Wasco MJ et al. Urothelial carcinoma with divergent histologic differentiation (mixed histologic features) predicts the presence of locally advanced bladder cancer when detected at transurethral resection. Urology. 2007;70(1):69-74.

17. Kim SP et al. The impact of squamous and glandular differentiation on survival after radical cystectomy for urothelial carcinoma. J Urol. 2012;188(2):405-9.

18. Nishiyama $\mathrm{H}$ et al. Clinical outcome of a large-scale multi-institutional retrospective study for locally advanced bladder cancer: a survey including 1131 patients treated during 1990-2000 in Japan. Eur Urol. 2004;45(2):176-81.

19. Monn MF et al. Primary choriocarcinoma of the bladder: a case report and review of literature. Clin Genitourin Cancer. 2017;15(2):18891.
20. Li G et al. Squamous differentiation in patients with superficial bladder urothelial carcinoma is associated with high risk of recurrence and poor survival. BMC Cancer. 2017;17(1):530.

21. Marks $P$ et al. The impact of variant histological differentiation on extranodal extension and survival in node positive bladder cancer treated with radical cystectomy. Surg Oncol. 2019;28:208-13.

22. Warrick JI. Clinical significance of histologic variants of bladder cancer. J Natl Compr Canc Netw. 2017;15(10):1268-74.

23. Shanks JH, Iczkowski KA. Divergent differentiation in urothelial carcinoma and other bladder cancer subtypes with selected mimics. Histopathology. 2009;54(7):885-900.

24. Solomon JP et al. Challenges in the diagnosis of urothelial carcinoma variants: can emerging molecular data complement pathology review? Urology. 2017;102:7-16.

25. Xu H et al. Impact of squamous and/or glandular differentiation on recurrence and progression following transurethral resection for non-muscle invasive urothelial carcinoma of bladder. Oncol Lett. 2017;14(3):3522-8.

26. Zhao G et al. Glandular differentiation in PT1 urothelial carcinoma of bladder predicts poor prognosis. Sci Rep. 2019;9(1):5323.

27. Samaratunga H, Delahunt B. Recently described and unusual variants of urothelial carcinoma of the urinary bladder. Pathology. 2012;44(5):40718.

28. Venyo AK. Nested variant of urothelial carcinoma. Adv Urol. 2014;2014:192720.

29. Wasco MJ et al. Nested variant of urothelial carcinoma: a clinicopathologic and immunohistochemical study of 30 pure and mixed cases. Hum Pathol. 
2010;41(2):163-71.

30. Linder BJ et al. Outcomes following radical cystectomy for nested variant of urothelial carcinoma: a matched cohort analysis. J Urol. 2013;189(5):1670-5.

31. Mally $A D$ et al. Clinical outcomes of patients with $\mathrm{T} 1$ nested variant of urothelial carcinoma compared to pure urothelial carcinoma of the bladder. Clin Genitourin Cancer. 2017:S1558-7673(17)30199-4.[Epub ahead of print].

32. Mitra AP et al. Implications of micropapillary urothelial carcinoma variant on prognosis following radical cystectomy: a multiinstitutional investigation. Urol Oncol. 2019;37(1):48-56.

33. Baumeister $\mathrm{P}$ et al. Histological variants in non-muscle invasive bladder cancer. Transl Androl Urol. 2019;8(1):34-8.

34. Abufaraj $\mathrm{M}$ et al. Micropapillary urothelial carcinoma of the bladder: a systematic review and metaanalysis of disease characteristics and treatment outcomes. Eur Urol. 2019;75(4):649-58.

35. Pantelides NM et al.

Lymphoepithelioma-like carcinoma of the urinary bladder: A case report and review of systemic treatment options. Urol ann. 2012;4(1):45-7.

36. Yang AW et al. Lymphoepitheliomalike, a variant of urothelial carcinoma of the urinary bladder: a case report and systematic review for optimal treatment modality for disease-free survival. BMC urology. 2017;17(1):34.
37. Singh NG et al. Lymphoepitheliomalike carcinoma of the urinary bladder: report of a rare case. Ann Saudi Med. 2009;29(6):478-81.

38. Mills SE et al. Lymphoepitheliomalike carcinoma of the uterine cervix. A distinctive, undifferentiated carcinoma with inflammatory stroma. Am J Surg Pathol. 1985;9(12):883-9.

39. Chou CW et al. Primary lymphoepithelioma-like carcinoma of the urinary bladder: case report and literature review. Urol Sci. 2012;23(4):125-8.

40. Jabbour $Y$ et al. Lymphoepitheliomalike carcinoma of the bladder: a case report of a rare and particular variant of urothelial carcinoma. Case Rep Urol. 2018:2018:7975454.

41. Nagai $T$ et al. Pure Iymphoepithelioma-like carcinoma originating from the urinary bladder. Case Rep Oncol. 2016;9(1)188-94.

42. Lopez-Beltran A, Cheng L. Histologic variants of urothelial carcinoma: differential diagnosis and clinical implications. Hum Pathol. 2006;37(11):1371-88.

43. Keck $B$ et al. The plasmacytoid carcinoma of the bladder--rare variant of aggressive urothelial carcinoma. Int J Cancer. 2011;129(2):346-54.

44. Dayyani F et al. Plasmacytoid urothelial carcinoma, a chemosensitive cancer with poor prognosis, and peritoneal carcinomatosis. J Urol. 2013;189(5):1656-61.

45. Chung $A D$ et al. Plasmacytoid urothelial carcinoma (PUC): imaging features with histopathological correlation. Can Urol Assoc J. 2017;11(1-2):E5O-7.

46. Lopez-Beltran A et al. Pathological variants of invasive bladder cancer according to their suggested clinical significance. BJU Int. 2008;101(3):27581.

47. Sui $W$ et al. Contemporary treatment patterns and outcomes of sarcomatoid bladder cancer. World J Urol. 2017;35(7):1055-61.

48. Bansal A et al. Sarcomatoid variant of urothelial carcinoma of the urinary bladder. J Cancer Res Ther. 2013;9(4):571-3.

49. Lopez-Beltran A et al. Pleomorphic giant cell carcinoma of the urinary bladder. Hum Pathol. 2009;40(10):1461-6.

50. 50. Samaratunga $\mathrm{H}$ et al Pleomorphic giant cell carcinoma of the urinary bladder: an extreme form of tumour de-differentiation. Histopathology. 2016;68(4):533-40.

51. Behzatoğlu K et al. Giant cell tumor-like lesion of the urinary bladder: a report of two cases and literature review; giant cell tumor or undifferentiated carcinoma? Diagn Pathol. 2009;4:48.

52. Lopez-Beltran A et al. Urothelial carcinoma of the bladder, lipid cell variant: clinicopathologic findings and $\mathrm{LOH}$ analysis. American J Surg Pathol. 2010;34(3):371-6. 